\title{
Gallium oxide as an insulating barrier for spin-dependent tunneling junctions
}

\author{
Zisen Li, Cornelis de Groot, and Jagadeesh H. Moodera ${ }^{a}$ \\ Francis Bitter Magnet Laboratory, Massachusetts Institute of Technology, Cambridge, Massachusetts 02139
}

(Received 14 August 2000; accepted for publication 29 September 2000)

\begin{abstract}
Spin-dependent tunneling has been shown to occur through $\mathrm{Ga}_{2} \mathrm{O}_{3}$ as the insulating tunnel barrier. Magnetic tunnel junctions of the type $\mathrm{Co} / \mathrm{Ga}_{2} \mathrm{O}_{3} / \mathrm{Ni}_{80} \mathrm{Fe}_{20}$ were prepared by oxidizing thin metal layer of $\mathrm{Ga}$ in oxygen plasma and characterized. The highest junction magnetoresistance observed was $18.2 \%$ at room temperature, increasing to $27.6 \%$ at $77 \mathrm{~K}$. The average barrier height was estimated to be about $2 \mathrm{eV}$, as opposed to over $3 \mathrm{eV}$ for junctions with $\mathrm{Al}_{2} \mathrm{O}_{3}$ barrier of comparable quality. Otherwise these junctions behave similar to those with $\mathrm{Al}_{2} \mathrm{O}_{3}$. This shows the feasibility of obtaining lower resistance junctions with $\mathrm{Ga}_{2} \mathrm{O}_{3}$ as the barrier for magnetic storage applications. (C) 2000 American Institute of Physics. [S0003-6951(00)04348-5]
\end{abstract}

Tunneling studies in magnetic tunnel junctions (MTJs) are being actively pursued in the last few years for their fundamental complexity as well as their application potential in data storage industry. ${ }^{1-3}$ It has been shown that the quality of the tunnel barriers plays a critical role in the success of spin tunneling in MTJs. ${ }^{1}$ Aluminum oxide is widely used as the insulating barrier since $\mathrm{Al}$ metal film can be reproducibly grown as ultrathin layer. ${ }^{4-7}$ The excellent insulating properties of ultrathin $\mathrm{Al}_{2} \mathrm{O}_{3}$ as well as its large forbidden gap, in general, lead to high resistance-area (RA) product, which is unfavorable from the application point of view. Recently there are reports wherein MTJs having lower RA values were obtained, using ultrathin $\mathrm{Al}_{2} \mathrm{O}_{3}$ barriers. ${ }^{6-8}$ Also, from the fundamental physics viewpoint, there are theoretical predictions and some experimental interpretations of barrier dependence of spin polarized tunneling. ${ }^{9}$ The motivation and necessity to study alternative tunnel barrier materials that show good spin tunneling properties is thus apparent. In this letter, our successful study of spin tunneling with $\mathrm{Ga}_{2} \mathrm{O}_{3}$ as tunnel barrier, in MTJs, is described.

$\mathrm{Ga}_{2} \mathrm{O}_{3}$ is an insulator with a forbidden gap of $4.8 \mathrm{eV}$ and is the only reported stable phase. ${ }^{10-12}$ However, there have been studies about the nonstoichiometric compound formation. $\mathrm{Ga}_{2} \mathrm{O}_{3-x}$ becomes $n$-type semiconductor due to oxygen vacancies, which act as shallow donors with activation energy of $30-40 \mathrm{meV}^{11} \mathrm{Ga}$ metal can be readily oxidized, relatively slower than $\mathrm{Al}_{2} \mathrm{O}_{3}$, having a heat of formation energy $(\mathrm{kJ} / \mathrm{mol})$ of 1080.2 compared to 1675.6 for $\mathrm{Al}_{2} \mathrm{O}_{3} .{ }^{10}$

MTJs were prepared in a high vacuum system (base pressure of $10^{-8}$ Torr) thermal evaporation system. ${ }^{1}$ Initially the $\mathrm{LN}_{2}$ cooled glass substrate was covered with a $1 \mathrm{~nm}$ thick $\mathrm{Si}$ seed layer, followed by an 8-nm-thick Co electrode of long strips. Shadow metal masks were used to define the film and the junction pattern. A gallium film with the thickness ranging from 0.4 to $1.8 \mathrm{~nm}$ was deposited to cover the entire Co layer. After the substrate was warmed to room temperature (RT) the Ga layer was oxidized in oxygen glow discharge plasma at a pressure of 80 mTorr and a dc voltage of

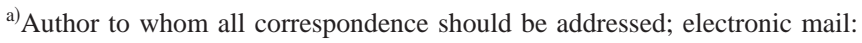
moodera@mit.edu
}

$1.6-1.8 \mathrm{kV}$ for varying time $(5-110 \mathrm{~s})$. After pumping down again, cross strips of the top electrode $\mathrm{Ni}_{80} \mathrm{Fe}_{20}, 10 \mathrm{~nm}$ thick, were deposited at RT. An overall protective layer of $1 \mathrm{~nm}$ thick Al film was deposited to complete the structure. In each run a total of 72 cross geometry planar junctions were prepared with 12 junctions of a given type.

The resulting junctions, $\mathrm{Co} / \mathrm{Ga}_{2} \mathrm{O}_{3} / \mathrm{Ni}_{80} \mathrm{Fe}_{20}$ of junction area $6 \times 10^{-8} \mathrm{~m}^{2}$, showed resistance $\left(R_{J}\right)$ in the range of tens of $\Omega$ to tens of $\mathrm{k} \Omega$, depending on the Ga layer thickness and the oxidation condition-junctions having the thinnest barrier, as expected, showing the lowest $R_{J}$. Junction magnetoresistance (JMR) was measured by four terminal dc technique both at 295 and $77 \mathrm{~K}$. In this letter we present data only for junctions having thicker Ga layers and $R_{J}$ in the $\mathrm{k} \Omega$ range. The best value of JMR (defined as the ratio of the change in $R_{J}$ in a magnetic field $H$ to the highest $R_{J}$ in $H$ ) was observed for Ga thickness of about 1.3-1.4 nm, oxidized for $80 \mathrm{~s}$.

The variation of resistance as a function of applied field for a junction formed with $1.4 \mathrm{~nm}$ Ga film barrier is shown in Fig. 1. At room temperature $R_{J}$ and JMR are $14.7 \mathrm{k} \Omega$ and $15.1 \%$, increasing to $19.2 \mathrm{k} \Omega$ and $24.6 \%$ upon cooling to 77 $\mathrm{K}$. Increase in $R_{J}$ and $\mathrm{JMR}$ was commonly observed upon cooling, whereas those junctions with either very thin or very

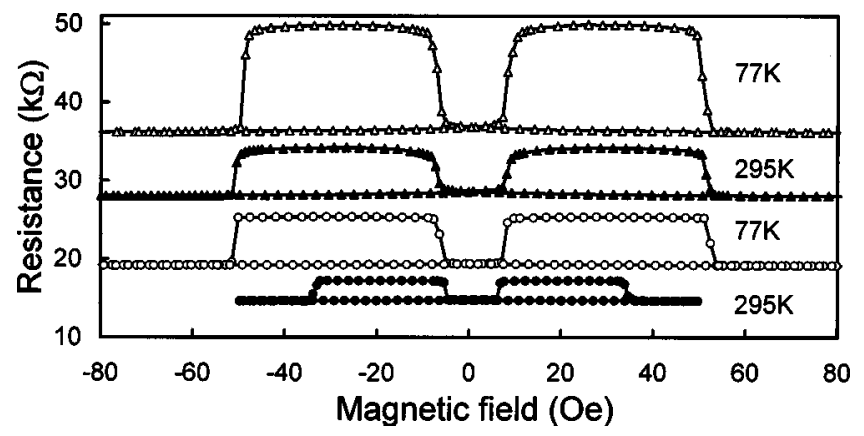

FIG. 1. Resistance vs applied magnetic field for a $\mathrm{Co} / \mathrm{Ga}_{2} \mathrm{O}_{3} / \mathrm{Ni}_{80} \mathrm{Fe}_{20}$ junction (with $1.4 \mathrm{~nm}$ Ga film oxidized) both as-grown (open and solid circle) and after $1 \mathrm{~h}$ vacuum annealing at $140{ }^{\circ} \mathrm{C}$ (open and solid triangle). JMR increased from $15.1 \%(295 \mathrm{~K})$ to $24.6 \%(77 \mathrm{~K})$ for as-grown junctions whereas after annealing the corresponding values were $18.2 \%$ and $27.6 \%$, respectively. 


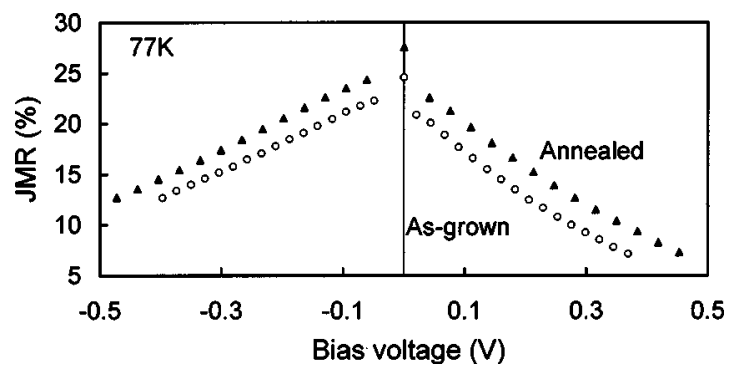

FIG. 2. Bias dependence of JMR for the same junction as in Fig. 1, before and after annealing.

thick Ga layer oxidized for a short time, showed significantly less JMR. (As an example, avoiding the current crowding effects, ${ }^{13}$ the JMR value for a junction with $0.7 \mathrm{~nm}$ thick $\mathrm{Ga}$ film was $9 \%$ and $13 \%$ at 295 and $77 \mathrm{~K}$, respectively. In this low thickness range of $\mathrm{Ga}$, further studies on ultrasmall area junctions with careful oxidation are needed to have optimum and reliable JMR values.) Such was also the case with earlier studies of $\mathrm{Al}_{2} \mathrm{O}_{3}$ barriers. ${ }^{5,7}$ Some of the junctions were annealed in vacuum $\left(10^{-6}\right.$ Torr $)$ at $140^{\circ} \mathrm{C}$ for $1 \mathrm{~h}$. The effect of such annealing is also shown in Fig. 1. Both $R_{J}$ and JMR increased after annealing, to $28.1 \mathrm{k} \Omega$ and $18.2 \%$, respectively, at $295 \mathrm{~K}$ and the corresponding values at $77 \mathrm{~K}$ were $36.2 \mathrm{k} \Omega$ and $27.6 \%$. The increase of JMR and $R_{J}$ values shows that during annealing the barrier quality has improved, similar to the case of $\mathrm{Al}_{2} \mathrm{O}_{3}$ barrier junctions. ${ }^{7,8,14-16}$ However, the annealing conditions were not optimized, whereas further studies can be expected to show better results.

The dc bias dependencies of JMR at $77 \mathrm{~K}$ for the above junction in the as-grown state and after annealing are shown in Fig. 2. The JMR decreases noticeably as the dc bias increases, and it is asymmetric. This is the case before as well as after annealing, although the JMR values are consistently higher at all $V_{\mathrm{dc}}$ for the annealed junction. Although the decrease in JMR with $V_{\mathrm{dc}}$ is significant, it is similar to initial results on $\mathrm{Al}_{2} \mathrm{O}_{3}$ barriers. ${ }^{17,18}$ In the latter case, there has been a steady improvement - the dc bias, at which JMR reduces to half its zero bias value, reaching $>0.5 \mathrm{~V}$, as the junction quality improved. ${ }^{7}$ The bias dependence has been addressed before, although not well understood. ${ }^{1,17,19,20}$

Shown in Fig. 3 are tunnel conductances as a function of dc bias at $77 \mathrm{~K}$. The conductance curves are asymmetric in the two bias directions and two orientations of relative magnetization. Notice also the broad bumpy feature at around $100 \mathrm{mV}$, a feature always seen for junctions when one of the electrodes is either Permalloy or $\mathrm{Ni}$ and with $\mathrm{Al}_{2} \mathrm{O}_{3}$ barrier. ${ }^{17}$

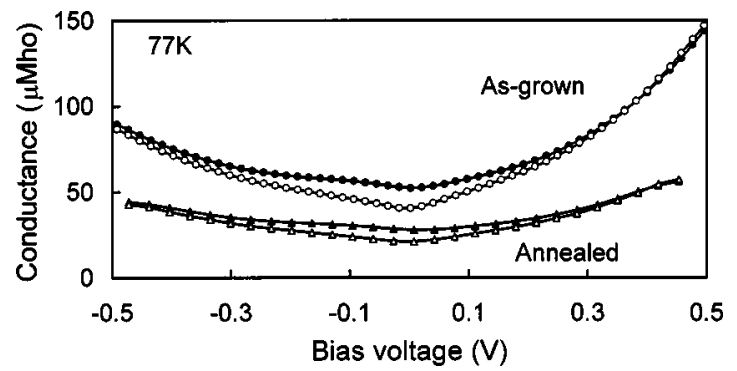

FIG. 3. Dynamic conductance as a function of de bias for parallel and antiparallel orientation of magnetizations of electrodes for the same junction as in Fig. 1. The lines are guide to the eye.

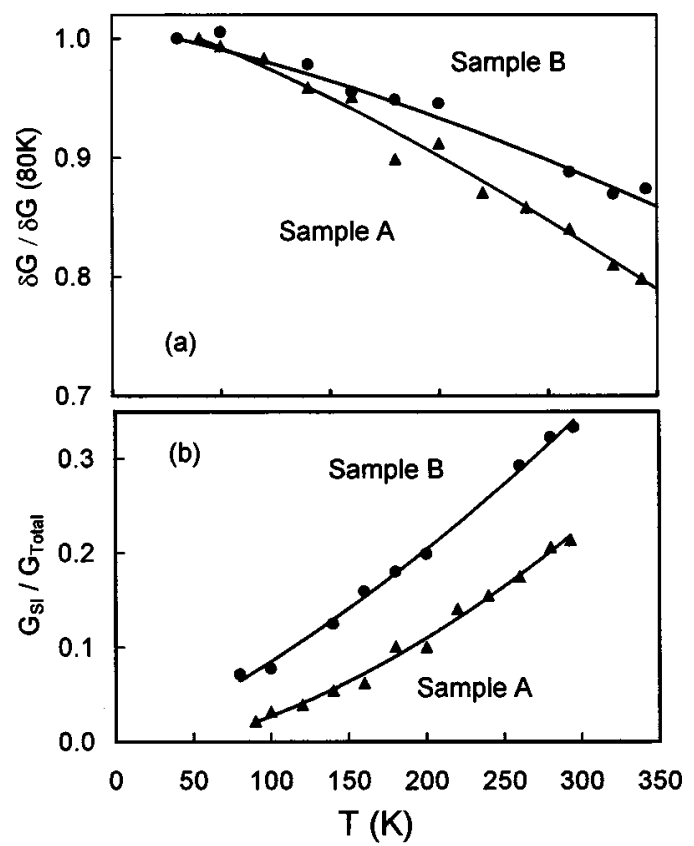

FIG. 4. Temperature dependence of (a) normalized spin dependant part of the conductance $\delta G$, and (b) relative contribution of spin-independent conductance $G_{\mathrm{SI}}$ normalized to total conductance. The junctions are $\mathrm{Co} / \mathrm{Ga}_{2} \mathrm{O}_{3} / \mathrm{Ni}_{80} \mathrm{Fe}_{20}$ formed with $1.4 \mathrm{~nm}$ Ga oxidized for $90 \mathrm{~s}$ (sample A) and $80 \mathrm{~s}$ (sample B). Solid lines are fits to the data.

Fitting the conductance versus bias data to Brinkmann's formula, ${ }^{21}$ the estimated average barrier height $(\phi)$ and thickness (s) are $1.9(2.3) \mathrm{eV}$ and $1.66(1.60) \mathrm{nm}$, respectively, for as-grown (annealed) junction. Barrier asymmetry reduced upon annealing the junction. ${ }^{7}$ The barrier heights obtained for various junctions were on the order or less than $2 \mathrm{eV}$. In comparison, similar quality junctions with $\mathrm{Al}_{2} \mathrm{O}_{3}$ barrier have shown $\phi>3 \mathrm{eV} .^{7,17}$ This is consistent with the fact that the forbidden energy gap for $\mathrm{Al}_{2} \mathrm{O}_{3}$ is higher than that for $\mathrm{Ga}_{2} \mathrm{O}_{3} \cdot{ }^{22}$ A lower barrier height increases the possibility for obtaining MTJ devices with lower RA product than with $\mathrm{Al}_{2} \mathrm{O}_{3}$ barriers, due to the exponential dependence of tunnel current on the barrier height. ${ }^{21}$ However, it should be noted that if the $\mathrm{Ga}_{2} \mathrm{O}_{3}$ were nonstoichiometric, possibly creating extra tunneling channels via defect states in the barrier, it would lead to an estimation of lower $\phi$. It was observed that even in the case of unannealed junctions, cooling to $1 \mathrm{~K}$ showed only a marginal improvement $(2 \%-3 \%)$ in the JMR values from their $77 \mathrm{~K}$ values, similar to the behavior of junctions with $\mathrm{Al}_{2} \mathrm{O}_{3}$ barrier. This indicates that the barriers are relatively free of defects. The increase of $\phi$ upon annealing can be partly attributed to a reduction of defects in $\mathrm{Ga}_{2} \mathrm{O}_{3}$ barrier, in addition to a possible sharpening of the ferromagnet/insulator interfaces. ${ }^{14,15}$

In order to understand the role of such defects in the barrier on spin tunneling, detailed temperature dependencies of JMR and $R_{J}$ were measured from 295 to $80 \mathrm{~K}$, for a number of samples. Based on our method of analysis as discussed in detail in an earlier publication, ${ }^{23}$ the conductance data were separated into spin dependent $(G)$ and spin independent $\left(G_{\mathrm{SI}}\right)$ parts. This is shown in Fig. 4 for two junctions. The normalized magnetoconductance $\delta G / \delta G(80 \mathrm{~K})$ (which is proportional to the product of spin polarization in the FMs) decreases as the temperature increases due to the 
decreasing spin polarization of the ferromagnet as $T$ increases. $^{23}$ In Fig. 4(a) the lines are fits to Julliere's model with the temperature dependence of spin polarization assumed to be similar to the surface magnetization temperature dependence. ${ }^{17}$ Details can be found in Ref. 23 .

Following our earlier method of analysis, for the case of the spin independent part, fitting the $G_{\mathrm{SI}}$ vs $T$ data to Glazman and Matveev model, $G_{\mathrm{SI}}(T) \propto T^{\gamma}$ [see the lines in Fig. 4(b)] gives a $\gamma$ of 2.11 and 1.51 for samples $\mathrm{A}$ and $\mathrm{B}$, respectively. Here $\gamma=N-[2 /(N+1)]$ and $N$ being the number of localized states through which electron hopping takes place. $^{24}$ Thus, the $\gamma$ values (leading to $N>2$ ) obtained can be attributed to the participation of more than two localized states during tunneling through the $\mathrm{Ga}_{2} \mathrm{O}_{3}$ barriers. This can give rise to multistep tunneling via these states with the involvement of phonons, thus influencing the spin-tunneling phenomenon. As a consequence, the spin independent contribution to the tunnel conductance increases at higher temperatures. The increase is larger for sample A. These two samples were prepared similarly except that for sample A, the glow discharge time was about $10 \%$ longer compared to sample B. It can be seen that over oxidation leads to a more defective barrier, as also seen earlier with $\mathrm{Al}_{2} \mathrm{O}_{3}$ barrier. ${ }^{7}$ Recently, the effect of states in the barrier on the spin dependent tunneling has been discussed. ${ }^{20,25}$

In summary, $\mathrm{Ga}_{2} \mathrm{O}_{3}$ has been used as an insulating barrier in the magnetic tunnel junctions resulting in a large junction magnetoresistance. The characteristics of this tunnel barrier are as good as the $\mathrm{Al}_{2} \mathrm{O}_{3}$ barrier. The observed lower barrier height for $\mathrm{Ga}_{2} \mathrm{O}_{3}$ as compared to a similar quality junction with $\mathrm{Al}_{2} \mathrm{O}_{3}$ barrier makes $\mathrm{Ga}_{2} \mathrm{O}_{3}$ advantageous for applications where low RA product is desirable.

It is a pleasure to thank undergraduates K. K. Wannomae and E. M. Lai for their assistance in this research. This work is supported by NSF (9730908) and ONR (0014-92-J1847) Grants. Zisen Li is supported by the TDK Research Award of J.H.M.

${ }^{1}$ J. S. Moodera, J. Nassar, and G. Mathon, Annu. Rev. Mater. Sci. 29, 381 (1999).

${ }^{2}$ W. J. Gallagher, S. S. P. Parkin, Y. Lu, X. P. Bian, A. Marley, K. P. Roche, R. A. Altman, S. A. Rishton, C. Jahnes, T. M. Shaw, and G. Xiao, J. Appl. Phys. 81, 3741 (1997).
${ }^{3}$ J. Zhang, Data Storage 31, (1998).

${ }^{4}$ J. S. Moodera, L. R. Kinder, T. M. Wong, and R. Meservey, Phys. Rev. Lett. 74, 3273 (1995).

${ }^{5}$ J. S. Moodera, E. F. Gallagher, K. Robinson, and J. Nowak, Appl. Phys. Lett. 70, 3050 (1997).

${ }^{6}$ T. Mitsuzuka, K. Matsuda, A. Kamijo, and H. Tsuge, J. Appl. Phys. 85, 5807 (1999).

${ }^{7}$ M. Covington, J. Nowak, and D. Song, Appl. Phys. Lett. 76, 3965 (2000).

${ }^{8}$ J. J. Sun, K. Shimazawa, N. Kasahara, K. Sato, S. Saruki, T. Kagami, O. Redon, S. Araki, H. Morita, and M. Matsuzaki, Appl. Phys. Lett. 76, 2424 (2000).

${ }^{9}$ E. Yu. Tsymbal and D. G. Pettifor, J. Phys.: Condens. Matter 9, L411 (1997); J. M. MacLaren, X. G. Zhang, and W. H. Butler, Phys. Rev. B 56, 11827 (1997); D. Nguyen-Manh, E. Y. Tsymbal, D. G. Pettifor, C. Arcangeli, R. Tank, O. K. Andersen, and A. Pasturel, Mater. Res. Soc. Symp. Proc. 492, 319 (1998); P. K. de Boer, G. A. de Wijs, and R. A. de Groot, Phys. Rev. B 58, 15422 (1998); J. M. De Teresa, A. Barthelemy, A. Fert, J. P. Contour, R. Lyonnet, F. Montaigne, P. Seneor, and A. Vaures, Phys. Rev. Lett. 82, 4288 (1999); M. Sharma, S. X. Wang, and J. Nickel, ibid. 82, 616 (1999).

${ }^{10}$ H. H. Tippins, Phys. Rev. 140, A316 (1965); See, also, The Oxide Handbook, edited by G. V. Samsonov (IFI/Plenum, New York, 1973).

${ }^{11}$ L. Binet, D. Gourier, and C. Minot, J. Solid State Chem. 113, 420 (1994).

${ }^{12}$ G. Schmitz, P. Gassmann, and R. Franchy, Surf. Sci. 397, 339 (1998).

${ }^{13}$ J. S. Moodera, L. R. Kinder, J. Nowak, P. LeClair, and R. Meservey, Appl. Phys. Lett. 69, 708 (1997); R. J. M. van de Veerdonk, J. Nowak, R. Meservey, J. S. Moodera, and W. J. M. deJonge, ibid. 71, 2839 (1997).

${ }^{14}$ J. Schmalhorst, H. Brückl, G. Reiss, M. Vieth, G. Gieres, and J. Wecker, J. Appl. Phys. 87, 5191 (2000).

${ }^{15}$ R. C. Sousa, J. J. Sun, V. Soares, P. P. Freitas, A. Kling, M. F. da Silva, and J. C. Soares, Appl. Phys. Lett. 73, 3288 (1998); S. Cardoso, P. P. Freitas, C. de Jesus, and J. C. Soares, J. Appl. Phys. 87, 6058 (2000).

${ }^{16}$ S. S. P. Parkin, K. P. Roche, M. G. Samant, P. M. Rice, R. B. Beyers, R. E. Scheuerlein, E. J. Sullivan, S. L. Brown, J. Bucchigano, D. W. Abraham, Y. Lu, M. Rooks, P. L. Trouilloud, R. A. Wanner, and W. J. Gallagher, J. Appl. Phys. 85, 5828 (1999).

${ }^{17}$ J. S. Moodera, J. Nowak, and R. J. van de Veerdonk, Phys. Rev. Lett. 80, 2941 (1998)

${ }^{18}$ Y. Lu, X. W. Li, G. Xiao, R. A. Altman, W. J. Gallagher, A. Marley, K. Roche, and S. S. P. Parkin, J. Appl. Phys. 83, 6515 (1998).

${ }^{19}$ S. Zhang, P. M. Levy, A. C. Marley, and S. S. P. Parkin, Phys. Rev. Lett. 79, 3744 (1997).

${ }^{20}$ A. M. Bratkovski, Phys. Rev. B 56, 2344 (1997); Appl. Phys. Lett. 72, 2334 (1998)

${ }^{21}$ W. F. Brinkman, R. C. Dynes, and J. M. Rowell, J. Appl. Phys. 41, 1915 (1970)

${ }^{22}$ A. M. Goodman, J. Appl. Phys. 41, 2176 (1970); F. Di Quarto, C. Gentile, S. Piazzo, and C. Sunseri, J. Electrochem. Soc. 138, 1856 (1991); see also, T. D. Burleigh, Mater. Sci. Forum 185-188, 447 (1995).

${ }^{23}$ C. H. Shang, J. Nowak, R. Jansen, and J. S. Moodera, Phys. Rev. B 58, R2917 (1998).

${ }^{24}$ L. I. Glatzman and K. A. Matveev, Sov. Phys. JETP 67, 1276 (1988).

${ }^{25}$ R. Jansen and J. S. Moodera, Phys. Rev. B 61, 9047 (2000). 